\title{
Soret and Hall effects on unsteady MHD free convection flow of radiating and chemically reactive fluid past a moving vertical plate with ramped temperature in rotating system
}

\author{
G. S. Seth*, B. Kumbhakar, S. Sarkar \\ Department of Applied Mathematics, Indian School of Mines, Dhanbad-826004, INDIA \\ *Corresponding Author: e-mail: gsseth_ism@yahoo.com
}

\begin{abstract}
Investigation of Soret and Hall effects on unsteady MHD free convection heat and mass transfer flow of a viscous, incompressible, electrically conducting and optically thick radiating fluid past an impulsively moving infinite vertical plate with ramped temperature through a uniform porous medium in a rotating system in the presence of first order chemical reaction is carried out. Exact analytical solution of the governing equations for fluid velocity, fluid temperature and species concentration subject to appropriate initial and boundary conditions is obtained using Laplace transform technique. Expressions for shear stress, rate of heat transfer and rate of mass transfer at the plate are derived for both ramped temperature and isothermal plates. The numerical values of primary and secondary fluid velocities, fluid temperature and species concentration are displayed graphically whereas those of shear stress and rate of mass transfer at the plate are presented in tabular form for various values of pertinent flow parameters.
\end{abstract}

Keywords: Magnetohydrodynamics (MHD), Hall current, chemical reaction, Soret effect, Ramped wall temperature.

DOI: http://dx.doi.org/10.4314/ijest.v7i2.8

\section{Introduction}

Investigation of unsteady hydromagnetic natural convection heat and mass transfer flow of a viscous, incompressible and electrically conducting fluid past solid bodies with different geometries embedded in porous and non-porous media has remained the focus of extensive research during past few decades due to its varied and wide range of applications in science and engineering such as boundary layer control, geothermal energy extraction, enhanced recovery of petroleum products and gases, metallurgy and controlled thermo-nuclear reactors etc. Processes involving heat and mass transfer flow through porous media are encountered in crystal formation, in reservoir engineering, in the study of the dynamics of hot and salty springs of a sea, grain storage, high performance insulation for buildings, migration of moisture through the air contained in fibrous and granular insulations, heat exchange between soil and atmosphere, sensible heat storage beds, geothermal energy systems etc. Keeping in mind the importance of such applications, Jha (1991), Kamel (2001), Ibrahim et al. (2004), Das et al. (2009), Eldabe et al. (2011) investigated unsteady hydromagnetic natural convection heat and mass transfer flow past a vertical plate in porous media considering different aspects of the problem.

It is well known that the effect of thermal radiation on hydromagnetic natural convection flow plays a significant role in many scientific and engineering processes such as casting and levitation, thermo-nuclear fusion, turbine blade heat transfer, furnace design, glass production, solar power technology, propulsion systems of aircrafts, missiles, satellites and space vehicles etc. It may be mentioned here that, unlike convection/conduction problems, the governing equations for fluid flow problems considering the effects of thermal radiation become quite complicated and hence many difficulties arise while solving such equations. So, a reasonable approximation to the radiative heat flux term is considered while solving those equations. A detailed explanation about radiative heat transfer may be found in the well documented text book by Sparrow and Cess (1966). Looking into the above 
mentioned facts, Takhar et al. (1996) investigated the effects of radiation on free convective flow along a semi-infinite vertical plate in the presence of transverse magnetic field. Azzam (2002) examined radiation effects on the MHD mixed convective flow of an optically thick fluid past a semi-infinite moving vertical plate considering Rosseland approximation to the radiative heat flux term. Ogulu and Makinde (2008) analyzed unsteady hydromagnetic free convection flow of a dissipative and radiative fluid past a vertical plate with constant heat flux. Suneetha et al. (2009) studied effects of thermal radiation on MHD natural convection heat and mass transfer flow of a viscous, incompressible and gray absorbing-emitting fluid past an impulsively started moving vertical plate with viscous dissipation. Mahmoud (2009) described thermal radiation effect on unsteady MHD free convection flow past a vertical plate with temperature dependent viscosity.

Moreover, study of heat and mass transfer flow in the presence of chemical reaction has received considerable attention due to its several practical applications in chemical and hydrometallurgical industries. Usually, chemical reactions are of two types, homogeneous and heterogeneous. A chemical reaction is said to be heterogeneous or homogenous depending on whether they occur at an interface or as a single phase volume reaction. A homogenous reaction occurs uniformly throughout the given phase, whereas a heterogeneous reaction takes place in a restricted region or within the boundary of a phase. Some of the numerous important applications of heat and mass transfer flow with chemical reaction can be found in catalytic chemical reactors, food processing, polymer production, manufacture of ceramics and glassware, smelting, undergoing exothermic or endothermic chemical reaction etc. Keeping in mind the significance of such study, several researchers investigated hydromagnetic free convection heat and mass transfer flow of a viscous, incompressible and electrically conducting fluid past a vertical plate in the presence of first order chemical reaction under different conditions. Some of the relevant research studies are due to Ibrahim et al. (2008), Mohamed and Abo-Dahab (2009), Singh and Kumar (2010), Pal and Talukdar (2011), Osman et al. (2011) and Barik and Dash (2014).

Hydromagnetic natural convection heat and mass transfer flow in a rotating system is of great importance because of its numerous applications in science and engineering such as geophysics, astrophysics, meteorology, oceanography, aeronautics, petrochemical engineering etc. This prompted several researchers to do a voluminous amount of research work on unsteady hydromagnetic heat and mass transfer flow of a viscous, incompressible and electrically conducting fluid past a vertical plate in a rotating system under different conditions. Mention may be made of the research studies of Tokis (1988), Nanousis (1992), Mbeledogu and Ogulu (2007) and Ferdows et al. (2008). Bakr (2011) studied effect of chemical reaction on MHD free convection heat and mass transfer flow of a micropolar fluid past an oscillatory moving vertical porous plate in the presence of a constant heat source in a rotating system. Das (2011) analyzed combined effect of chemical reaction and thermal radiation on hydromagnetic free convection heat and mass transfer flow of a micropolar fluid through a porous medium past an oscillating semi-infinite porous plate with constant heat source in a rotating frame of reference. Recently, Barik et al. (2014) investigated unsteady MHD free convective flow and mass transfer of a viscous, incompressible and electrically conducting fluid past an infinite vertical porous surface through a porous medium in a rotating system with first order chemical reaction taking a fluctuating heat source/sink into account.

It is worthy to note that, in an ionized fluid, where density is low and/or magnetic field is strong, the effects of Hall current becomes significant as described by Sutton and Sherman (1965). In fact, Hall current plays a key role in determining the flowfeatures of the fluid flow problems because it induces secondary flow in the flow-field which is also the characteristics of Coriolis force. Therefore, it seems to be significant to compare and contrast the simultaneous effects of these two agencies on the fluid flow problems. Hall current finds applications in many scientific and engineering devices, viz. MHD power generators, Hall current accelerators, nuclear power reactors, magnetometers, underground energy storage system, Hall effect sensors, spacecraft propulsion etc. Taking into consideration the importance of combined effects of Hall current and rotation, Alam and Sattar (2000) investigated unsteady hydromagnetic free convection heat and mass transfer flow with Hall effects in a rotating system in the presence of viscous dissipation and Joule heating. Recently, Seth et al. (2014) studied effects of Hall current and rotation on unsteady hydromagnetic natural convection heat and mass transfer flow of an optically thick radiating fluid past an infinite vertical plate through porous medium.

However, in all the above investigations, the solution for fluid flow problems is obtained by assuming conditions for the fluid temperature at the plate as continuous and well defined. But, there are several physical situations where the temperature at the bounding surface may require non-uniform or arbitrary wall conditions. In fact, there may be a step discontinuity in the wall temperature (Lee and Yovanovich (1991)) or there may be ramped wall temperature (Chandran et al. (2005), Seth et al. (2011)). For example, in the fabrication of thin-film photovoltaic devices ramped wall temperatures are employed to achieve a specific finish of the system (Malhotra et al. (2006)). Application of ramped type wall temperature is also found in nuclear heat transfer control, materials processing, turbine blade heat transfer, electronic circuits and sealed gas-filled enclosure heat transfer operations. Keeping in mind the importance of this fact Nandkeolyar et al. (2013) studied unsteady hydromagnetic free convective flow of a viscous, incompressible, electrically conducting and heat radiating fluid past a flat plate with ramped wall temperature in the presence of suction/blowing. Seth et al. (2013) investigated effect of radiation and rotation on unsteady hydromagnetic free convection flow of an electrically conducting fluid past an impulsively moving infinite vertical plate with ramped temperature embedded in a porous medium. Recently, Seth et al. (2015) analyzed unsteady hydromagnetic natural convection heat and mass transfer flow with Hall effects of a heat absorbing and optically thick radiating fluid past an accelerated moving vertical plate with ramped temperature and ramped surface concentration in the presence of chemical reaction through a porous medium. 
It is to be noted that in all the above research studies, Soret (or thermo-diffusion) effect assumed to be neglected. But, Soret effect becomes significant when there exists a density difference in the flow regime i.e. in the areas such as hydrology, petrology and geo-sciences. This effect has various important applications in isotope separation, in mixture between gases with very light molecular weight $\left(\mathrm{H}_{2}, \mathrm{He}\right)$ and of medium molecular weight $\left(\mathrm{N}_{2}\right.$, air) etc. Comprehensive information on the importance of Soret effect in convective transport in clear fluids can be found in the textbook of Eckert and Drake (1972). Looking into the importance of such effect, Jha and Singh (1990) analyzed Soret effect on unsteady free convection and mass transfer flow past an impulsively started infinite vertical plate. Kafoussias (1992) investigated Soret effect on unsteady hydromagnetic free convection heat and mass transfer past an infinite moving vertical plate for two cases of practical interest, namely, (i) impulsively started plate and (ii) uniformly accelerated plate. Reddy and Rao (2011) studied Soret effect on unsteady MHD free convection heat and mass transfer flow past an infinite vertical porous plate in the presence of Hall current, thermal radiation and a heat source. Pal and Talukdar (2012) described the influence of thermal radiation and first-order chemical reaction on unsteady MHD convective heat and mass transfer flow with Soret effect of a viscous, incompressible and electrically conducting fluid past a semi-infinite vertical flat plate in the presence oscillatory suction and heat source.

As per authors' knowledge, no attempt has been made to study unsteady hydromagnetic free convection heat and mass transfer flow past a vertical plate with ramped temperature taking Soret effect into account. Objective of the present investigation is to analyze unsteady hydromagnetic free convection heat and mass transfer flow with Soret and Hall effects of a viscous, incompressible, electrically conducting and optically thick radiating fluid past an impulsively moving infinite vertical plate with ramped temperature through a uniform porous medium in a rotating system in the presence of first order chemical reaction.

\section{Formulation of the problem and its solution}

Consider unsteady hydromagnetic free convection heat and mass transfer of flow with Soret and Hall effects of a viscous, incompressible, electrically conducting, chemically reactive and optically thick radiating fluid past an infinite moving vertical plate with ramped temperature through a uniform porous medium in a rotating system. Cartesian coordinate system $\left(x^{\prime}, y^{\prime}, z^{\prime}\right)$ is chosen in such a way that $x^{\prime}$-axis is along the vertical plate in upward direction, $y^{\prime}$-axis is normal to plane of the plate and directed into the fluid region and $z^{\prime}$-axis is perpendicular to $x^{\prime} y^{\prime}$ plane. A uniform transverse magnetic field $B_{0}$ is applied in a direction which is parallel to $y^{\prime}$-axis. The fluid and plate rotate in unison in anticlockwise direction with uniform angular velocity $\Omega$ about $y^{\prime}$-axis. Initially i.e. at time $t^{\prime} \leq 0$, both the fluid and plate are at rest and are maintained at uniform temperature $T_{\infty}^{\prime}$. Also species concentration at the surface of the plate as well as at every point within the fluid is maintained at uniform concentration $C_{\infty}^{\prime}$. At time $t^{\prime}>0$, plate starts moving in $x^{\prime}$ - direction with uniform velocity $U_{0}$ in its own plane. The temperature of the plate is raised to $T_{\infty}^{\prime}+\left(T_{w}^{\prime}-T_{\infty}^{\prime}\right) t^{\prime} / t_{0}$ when $0<t^{\prime} \leq t_{0}$, and is maintained at uniform temperature $T_{w}^{\prime}$ when $t^{\prime}>t_{0} \quad\left(t_{0}\right.$ being the characteristic time). Also, at time $t^{\prime}>0$, species concentration at the surface of the plate is raised to uniform species concentration $C_{w}^{\prime}$ and is maintained thereafter. It is assumed that there exists a homogeneous chemical reaction of first order with uniform rate $K_{r}^{\prime}$ between the diffusing species and the ambient fluid. Geometry of the problem is presented in Figure 1.

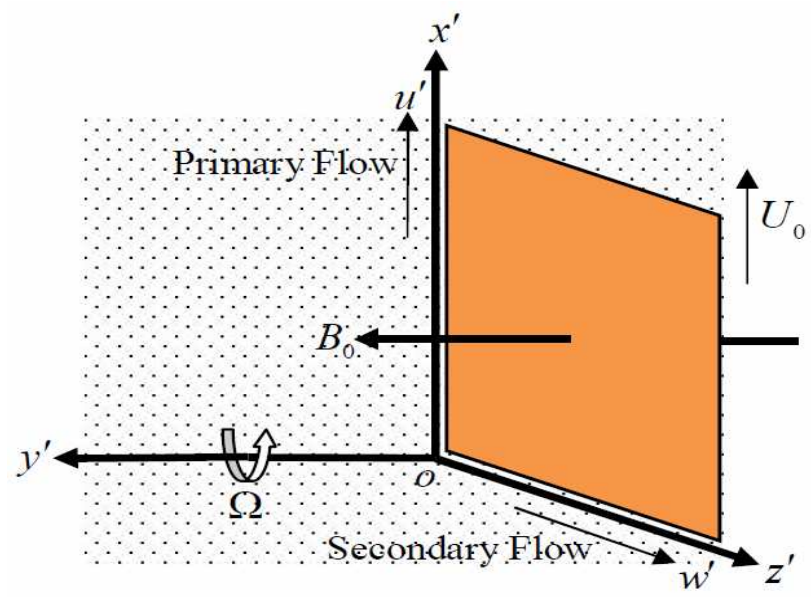

Figure 1 Geometry of the problem.

Since plate is of infinite extent in $x^{\prime}$ and $z^{\prime}$ directions and is electrically non-conducting, all physical quantities except pressure depend on $y^{\prime}$ and $t^{\prime}$ only. Also no applied or polarized voltages exist so that the effect of polarization of fluid is 
negligible. This corresponds to the case where no energy is added or extracted from the fluid by electrical means (Cramer and Pai, 1973). Further, it is also assumed that the induced magnetic field generated by fluid motion is negligible in comparison to the applied one. This assumption is justified because magnetic Reynolds number is very small for liquid metals and partially ionized fluids which are commonly used in industrial applications (Cramer and Pai, 1973).

Keeping in view the assumptions made above, governing equations for unsteady hydromagnetic natural convection heat and mass flow transfer of a viscous, incompressible, electrically conducting and optically thick radiating fluid through a uniform porous medium in a rotating frame of reference taking Hall current, Soret effect and first order homogeneous chemical reaction into account, under Boussinesq approximation, are given by

$$
\begin{aligned}
& \frac{\partial u^{\prime}}{\partial t^{\prime}}+2 \Omega w^{\prime}=v \frac{\partial^{2} u^{\prime}}{\partial y^{\prime 2}}-\frac{\sigma B_{0}^{2}}{\rho\left(1+m^{2}\right)}\left(u^{\prime}+m w^{\prime}\right)-\frac{v}{K_{1}^{\prime}} u^{\prime}+g \beta^{\prime}\left(T^{\prime}-T_{\infty}^{\prime}\right)+g \beta^{*}\left(C^{\prime}-C_{\infty}^{\prime}\right), \\
& \frac{\partial w^{\prime}}{\partial t^{\prime}}-2 \Omega u^{\prime}=v \frac{\partial^{2} w^{\prime}}{\partial y^{\prime 2}}+\frac{\sigma B_{0}^{2}}{\rho\left(1+m^{2}\right)}\left(m u^{\prime}-w^{\prime}\right)-\frac{v}{K_{1}^{\prime}} w^{\prime}, \\
& \frac{\partial T^{\prime}}{\partial t^{\prime}}=\frac{k}{\rho c_{p}} \frac{\partial^{2} T^{\prime}}{\partial y^{\prime 2}}-\frac{1}{\rho c_{p}} \frac{\partial q_{r}^{\prime}}{\partial y^{\prime}}, \\
& \frac{\partial C^{\prime}}{\partial t^{\prime}}=D_{m} \frac{\partial^{2} C^{\prime}}{\partial y^{\prime 2}}+\frac{D_{m} k_{T}}{T_{m}} \frac{\partial^{2} T^{\prime}}{\partial y^{\prime 2}}-K_{r}^{\prime}\left(C^{\prime}-C_{\infty}^{\prime}\right),
\end{aligned}
$$

where $u^{\prime}, w^{\prime}, T^{\prime}, C^{\prime}, v, \rho, \sigma, m=\omega_{e} \tau_{e}, \omega_{e}, \tau_{e}, g, \beta^{\prime}, \beta^{*}, c_{p}, D_{m}, k, k_{T}, K_{1}^{\prime}, K_{r}^{\prime}, q_{r}^{\prime}$ and $T_{m}$ are, respectively, fluid velocity in $x^{\prime}$-direction, fluid velocity in $z^{\prime}$-direction, fluid temperature, species concentration, kinematic coefficient of viscosity, fluid density, electrical conductivity, Hall current parameter, cyclotron frequency, electron collision time, acceleration due to gravity, coefficient of thermal expansion, coefficient of expansion of species concentration, specific heat at constant pressure, molecular mass diffusivity, thermal diffusivity, thermal conductivity of the fluid, thermal diffusion ratio, permeability of the porous medium, chemical reaction coefficient, radiative heat flux and mean fluid temperature.

Initial and boundary conditions for the fluid flow problem are specified below

$$
\begin{array}{ll}
t^{\prime} \leq 0 & : u^{\prime}=0, \quad w^{\prime}=0, \quad C^{\prime}=C_{\infty}^{\prime}, \quad T^{\prime}=T_{\infty}^{\prime} \text { for all } y^{\prime} \geq 0, \\
t^{\prime}>0 & : u^{\prime}=U_{0}, \quad w^{\prime}=0, \quad C^{\prime}=C_{w}^{\prime}, \quad T^{\prime}= \begin{cases}T_{\infty}^{\prime}+\left(T_{w}^{\prime}-T_{\infty}^{\prime}\right) t^{\prime} / t_{0} & \text { at } y^{\prime}=0 \text { for } 0<t^{\prime} \leq t_{0}, \\
T_{w}^{\prime} & \text { at } y^{\prime}=0 \text { for } t^{\prime}>t_{0}\end{cases} \\
t^{\prime}>0 & : u^{\prime} \rightarrow 0, \quad w^{\prime} \rightarrow 0, \quad C^{\prime} \rightarrow C_{\infty}^{\prime}, \quad T^{\prime} \rightarrow T_{\infty}^{\prime} \text { as } y^{\prime} \rightarrow \infty .
\end{array}
$$

For an optically thick grey fluid, the radiative heat flux $q_{r}^{\prime}$ is approximated by Rosseland approximation as follows:

$q_{r}^{\prime}=-\frac{4 \sigma^{*}}{3 k^{*}} \frac{\partial T^{\prime 4}}{\partial y^{\prime}}$,

where $\sigma^{*}$ and $k^{*}$ are Stefan Boltzmann constant and mean absorption coefficient respectively.

It is assumed that, temperature difference between the fluid near the plate and the free-stream is very small so that $T^{\prime 4}$ may be expressed as a linear function of temperature $T^{\prime}$. Expanding $T^{\prime 4}$ in Taylor series about $T_{\infty}^{\prime}$ and neglecting the second and higher order terms, we obtain

$T^{\prime 4} \cong 4 T_{\infty}^{\prime 3} T^{\prime}-3 T_{\infty}^{\prime 4}$.

Using (6) and (7) in equation (3), we obtain

$$
\frac{\partial T^{\prime}}{\partial t^{\prime}}=\frac{k}{\rho c_{p}}\left(1+\frac{16 \sigma^{*} T_{\infty}^{\prime 3}}{3 k k^{*}}\right) \frac{\partial^{2} T^{\prime}}{\partial y^{\prime 2}} .
$$

In order to non-dimensionalize equations (1), (2), (4) and (8) along with initial and boundary conditions (5a) to (5c), we introduce the following non-dimensional variables and parameters: 


$$
\begin{aligned}
& \eta=y^{\prime} / U_{0} t_{0}, t=t^{\prime} / t_{0}, u=u^{\prime} / U_{0}, w=w^{\prime} / U_{0}, T=\left(T^{\prime}-T_{\infty}^{\prime}\right) /\left(T_{w}^{\prime}-T_{\infty}^{\prime}\right), C=\left(C^{\prime}-C_{\infty}^{\prime}\right) /\left(C_{w}^{\prime}-C_{\infty}^{\prime}\right), \\
& G_{r}=g \beta^{\prime} v\left(T_{w}^{\prime}-T_{\infty}^{\prime}\right) / U_{0}^{3}, G_{c}=g \beta^{*} v\left(C_{w}^{\prime}-C_{\infty}^{\prime}\right) / U_{0}^{3}, K^{2}=\Omega v / U_{0}^{2}, K_{1}=K_{1}^{\prime} U_{0}^{2} / v^{2}, K_{r}=K_{r}^{\prime} t_{0}, \\
& \left.M^{2}=\sigma B_{0}^{2} v / \rho U_{0}^{2}, N_{r}=16 \sigma^{*} T_{\infty}^{\prime 3} / 3 k k^{*}, P_{r}=\rho v c_{p} / k, S_{c}=v / D_{m}, S_{r}=\left(T_{w}^{\prime}-T_{\infty}^{\prime}\right) D_{m} k_{T} /\left(C_{w}^{\prime}-C_{\infty}^{\prime}\right) T_{m} v,\right\}
\end{aligned}
$$

where $G_{r}, G_{c}, K^{2}, K_{1}, K_{r}, M^{2}, N_{r}, P_{r}, S_{c}, S_{r}$ are thermal Grashof number, solutal Grashof number, rotation parameter, permeability parameter, chemical reaction parameter, magnetic parameter, radiation parameter, Prandtl number, Schmidt number and Soret number respectively.

With the help of (9), equations (1), (2), (4) and (8), in non-dimensional form, become

$$
\begin{aligned}
& \frac{\partial u}{\partial t}+2 K^{2} w=\frac{\partial^{2} u}{\partial \eta^{2}}-\frac{M^{2}}{1+m^{2}}(u+m w)-\frac{1}{K_{1}} u+G_{r} T+G_{c} C, \\
& \frac{\partial w}{\partial t}-2 K^{2} u=\frac{\partial^{2} w}{\partial \eta^{2}}+\frac{M^{2}}{1+m^{2}}(m u-w)-\frac{1}{K_{1}} w, \\
& \frac{\partial T}{\partial t}=\frac{1+N_{r}}{P_{r}} \frac{\partial^{2} T}{\partial \eta^{2}}, \\
& \frac{\partial C}{\partial t}=\frac{1}{S_{c}} \frac{\partial^{2} C}{\partial \eta^{2}}+S_{r} \frac{\partial^{2} T}{\partial \eta^{2}}-K_{r} C,
\end{aligned}
$$

Initial and boundary conditions (5a) to (5c), in non-dimensional form, are given by

$t \leq 0 \quad: u=0, \quad w=0, \quad C=0, \quad T=0 \quad$ for all $\eta \geq 0$,

$t>0 \quad: u=1, \quad w=0, \quad C=1, \quad T= \begin{cases}t & \text { at } \eta=0 \text { for } 0<t \leq 1, \\ 1 & \text { at } \eta=0 \text { for } t>1\end{cases}$

$t>0 \quad: u \rightarrow 0, w \rightarrow 0, C \rightarrow 0, T \rightarrow 0$ as $\eta \rightarrow \infty$,

In the above non-dimensional process, characteristic time $t_{0}$ is considered as $t_{0}=v / U_{0}^{2}$.

Equations (10) and (11) are combined and expressed in compact form as

$\frac{\partial F}{\partial t}+\left\{\frac{M^{2}(1-i m)}{1+m^{2}}+\frac{1}{K_{1}}-2 i K^{2}\right\} F=\frac{\partial^{2} F}{\partial \eta^{2}}+G_{r} T+G_{c} C$,

where $F(\eta, t)=u(\eta, t)+i w(\eta, t)$.

Initial and boundary conditions (14a)-(14c), in compact form, are given by

$t \leq 0 \quad: F=0, \quad C=0, \quad T=0 \quad$ for all $\eta \geq 0$,

$t>0 \quad: F=1, \quad C=1, \quad T= \begin{cases}t & \text { at } \eta=0 \text { for } 0<t \leq 1, \\ 1 & \text { at } \eta=0 \text { for } t>1\end{cases}$

$t>0 \quad: F \rightarrow 0, \quad C \rightarrow 0, T \rightarrow 0 \quad$ as $\eta \rightarrow \infty$,

Exact analytical solution for fluid temperature $T(\eta, t)$, species concentration $C(\eta, t)$ and fluid velocity $F(\eta, t)$ is obtained by solving equations (12), (13) and (15) subject to the initial and boundary conditions (17a) to (17c) using Laplace transform technique and are expressed in the following simplified form:

$$
\begin{aligned}
T(\eta, t)= & T_{0}(\eta, t)-H(t-1) T_{0}(\eta, t-1), \\
C(\eta, t)= & (1 / 2)\left[C_{0}(\eta, t)+\left(\lambda_{1} / \lambda_{2}\right)\left\{C_{1}(\eta, t)-H(t-1) C_{1}(\eta, t-1)\right\}\right], \\
F(\eta, t)= & (1 / 2)\left[F_{0}(\eta, t)-D_{1} F_{1}(\eta, t)+D_{2} F_{2}(\eta, t)+D_{3} F_{3}(\eta, t)+D_{4} F_{4}(\eta, t)\right. \\
& \left.+H(t-1)\left\{D_{1} F_{1}(\eta, t-1)-D_{2} F_{2}(\eta, t-1)-D_{4} F_{4}(\eta, t-1)\right\}\right],
\end{aligned}
$$


where

$$
\begin{aligned}
& T_{0}(\eta, t)=\left(t+\frac{\eta^{2} a}{2}\right) \operatorname{erfc}\left(\frac{\eta}{2} \sqrt{\frac{a}{t}}\right)-\sqrt{\frac{t a}{\pi}} \eta e^{-\frac{\eta^{2} a}{4 t}}, \\
& C_{0}(\eta, t)=f_{1}\left(\eta, t, S_{c}, 0, K_{r}\right) \text {, } \\
& C_{1}(\eta, t)=e^{\lambda_{2} t}\left\{f_{1}\left(\eta, t, S_{c}, \lambda_{2}, K_{r}\right)-f_{1}\left(\eta, t, a, \lambda_{2}, 0\right)\right\}-\left\{f_{1}\left(\eta, t, S_{c}, 0, K_{r}\right)-f_{1}(\eta, t, a, 0,0)\right\}, \\
& F_{0}(\eta, t)=f_{1}\left(\eta, t, 1, \lambda_{3}, 0\right) \text {, } \\
& F_{1}(\eta, t)=\frac{1}{\lambda_{2} \lambda_{4}\left(\lambda_{2}-\lambda_{4}\right)}\left[\lambda_{4} e^{\lambda_{2} t}\left\{f_{1}\left(\eta, t, 1, \lambda_{2}, \lambda_{3}\right)-f_{1}\left(\eta, t, a, \lambda_{2}, 0\right)\right\}-\lambda_{2} e^{\lambda_{4} t}\left\{f_{1}\left(\eta, t, 1, \lambda_{3}, \lambda_{4}\right)\right.\right. \\
& \left.\left.-f_{1}\left(\eta, t, a, 0, \lambda_{4}\right)\right\}+\left(\lambda_{2}-\lambda_{4}\right)\left\{f_{1}\left(\eta, t, 1, \lambda_{3}, 0\right)-f_{1}(\eta, t, a, 0,0)\right\}\right], \\
& F_{2}(\eta, t)=\frac{1}{\lambda_{4}^{2}}\left[e^{\lambda_{4} t}\left\{f_{1}\left(\eta, t, 1, \lambda_{3}, \lambda_{4}\right)-f_{1}\left(\eta, t, a, 0, \lambda_{4}\right)\right\}-\lambda_{4}\left\{f_{2}\left(\eta, t, \lambda_{3}, \lambda_{4}\right)-2 f_{3}\left(\eta, t, a, \lambda_{4}, \pi\right)\right\}\right], \\
& F_{3}(\eta, t)=\frac{1}{\lambda_{5}}\left[e^{\lambda_{5} t}\left\{f_{1}\left(\eta, t, 1, \lambda_{3}, \lambda_{5}\right)-f_{1}\left(\eta, t, S_{c}, K_{r}, \lambda_{5}\right)\right\}-\left\{f_{1}\left(\eta, t, 1, \lambda_{3}, 0\right)-f_{1}\left(\eta, t, S_{c}, K_{r}, 0\right)\right\}\right] \text {, } \\
& F_{4}(\eta, t)=\frac{1}{\lambda_{2} \lambda_{5}\left(\lambda_{2}-\lambda_{5}\right)}\left[\lambda_{5} e^{\lambda_{2} t}\left\{f_{1}\left(\eta, t, 1, \lambda_{2}, \lambda_{3}\right)-f_{1}\left(\eta, t, S_{c}, \lambda_{2}, K_{r}\right)\right\}-\lambda_{2} e^{\lambda_{5} t}\left\{f_{1}\left(\eta, t, 1, \lambda_{3}, \lambda_{5}\right)\right.\right. \\
& \left.\left.-f_{1}\left(\eta, t, S_{c}, K_{r}, \lambda_{5}\right)\right\}+\left(\lambda_{2}-\lambda_{5}\right)\left\{f_{1}\left(\eta, t, 1, \lambda_{3}, 0\right)-f_{1}\left(\eta, t, S_{c}, K_{r}, 0\right)\right\}\right], \\
& \lambda_{1}=\frac{S_{c} S_{r} a}{a-S_{c}}, \lambda_{2}=\frac{S_{c} K_{r}}{a-S_{c}}, \lambda_{3}=\frac{M^{2}(1-i m)}{1+m^{2}}+\frac{1}{K_{1}}-2 i K^{2}, \lambda_{4}=\frac{\lambda_{3}}{a-1}, \lambda_{5}=\frac{\lambda_{3}-S_{c} K_{r}}{S_{c}-1}, a=\frac{P_{r}}{1+N_{r}}, \\
& D_{1}=\frac{\lambda_{1} G_{c}}{a-1}, D_{2}=\frac{G_{r}}{a-1}, D_{3}=\frac{G_{c}}{S_{c}-1}, D_{4}=\frac{\lambda_{1} G_{c}}{S_{c}-1} \text {. }
\end{aligned}
$$

The expressions for $f_{i}, i=1,2,3$ are defined in Appendix.

\subsection{Solution in case of isothermal plate}

Solutions (18) to (20) represent the analytical solution for fluid temperature, species concentration and fluid velocity for unsteady hydromagnetic free convection heat and mass transfer flow with Hall and Soret effects of a viscous, incompressible, electrically conducting, chemically reactive and optically thick radiating fluid past an impulsively moving vertical plate with ramped temperature through a uniform porous medium in a rotating system. In order to highlight the influence of ramped temperature distribution within the plate on the flow-field, it may be justified to compare such a flow with the one past an impulsively moving vertical plate with uniform temperature. Keeping in view the assumptions made in Section 2, the solution for the fluid temperature, species concentration and fluid velocity for the flow past an impulsively moving isothermal vertical plate is obtained and presented in the following simplified form:

$$
\begin{aligned}
& T(\eta, t)=\operatorname{erfc}\left(\frac{\eta}{2} \sqrt{\frac{a}{t}}\right), \\
& C(\eta, t)=(1 / 2)\left[C_{0}(\eta, t)+\lambda_{1} C_{2}(\eta, t)\right], \\
& F(\eta, t)=(1 / 2)\left[G_{0}(\eta, t)-D_{1} G_{1}(\eta, t)+D_{2} G_{2}(\eta, t)+D_{3} G_{3}(\eta, t)+D_{4} G_{4}(\eta, t)\right],
\end{aligned}
$$

where

$$
\begin{aligned}
& C_{2}(\eta, t)=e^{\lambda_{2} t}\left\{f_{1}\left(\eta, t, S_{c}, \lambda_{2}, K_{r}\right)-f_{1}\left(\eta, t, a, \lambda_{2}, 0\right)\right\} \\
& G_{0}(\eta, t)=f_{1}\left(\eta, t, 1, \lambda_{3}, 0\right) \\
& G_{1}(\eta, t)=\frac{1}{\left(\lambda_{2}-\lambda_{4}\right)}\left[e^{\lambda_{2} t}\left\{f_{1}\left(\eta, t, 1, \lambda_{2}, \lambda_{3}\right)-f_{1}\left(\eta, t, a, \lambda_{2}, 0\right)\right\}-e^{\lambda_{4} t}\left\{f_{1}\left(\eta, t, 1, \lambda_{3}, \lambda_{4}\right)-f_{1}\left(\eta, t, a, 0, \lambda_{4}\right)\right\}\right] \\
& G_{2}(\eta, t)=\frac{1}{\lambda_{4}}\left[e^{\lambda_{4} t}\left\{f_{1}\left(\eta, t, 1, \lambda_{3}, \lambda_{4}\right)-f_{1}\left(\eta, t, a, 0, \lambda_{4}\right)\right\}-\left\{f_{1}\left(\eta, t, 1, \lambda_{3}, 0\right)-f_{1}(\eta, t, a, 0,0)\right\}\right]
\end{aligned}
$$




$$
\begin{aligned}
& G_{3}(\eta, t)=\frac{1}{\lambda_{5}}\left[e^{\lambda_{5} t}\left\{f_{1}\left(\eta, t, 1, \lambda_{3}, \lambda_{5}\right)-f_{1}\left(\eta, t, S_{c}, K_{r}, \lambda_{5}\right)\right\}-\left\{f_{1}\left(\eta, t, 1, \lambda_{3}, 0\right)-f_{1}\left(\eta, t, S_{c}, K_{r}, 0\right)\right\}\right] \\
& G_{4}(\eta, t)=\frac{1}{\left(\lambda_{2}-\lambda_{5}\right)}\left[e^{\lambda_{2} t}\left\{f_{1}\left(\eta, t, 1, \lambda_{2}, \lambda_{3}\right)-f_{1}\left(\eta, t, S_{c}, \lambda_{2}, K_{r}\right)\right\}-e^{\lambda_{5} t}\left\{f_{1}\left(\eta, t, 1, \lambda_{3}, \lambda_{5}\right)-f_{1}\left(\eta, t, S_{c}, K_{r}, \lambda_{5}\right)\right\}\right] .
\end{aligned}
$$

\subsection{Solution in case of unit Schmidt number}

It is observed from solutions (20) and (23) that the solution for fluid velocity for both ramped temperature and isothermal plates are not valid for the fluids with unit Schmidt number. Since Schmidt number represents the measure of the relative strength of viscosity to molecular diffusivity of the fluid, therefore, fluid flow problem with $S_{c}=1$ corresponds to those fluids for which both viscous and concentration boundary layer thicknesses are of same order of magnitude (Symeonidis et al., 2006). Substituting $S_{c}=1$ in equation (13) and following the same procedure as adopted earlier, exact solution for fluid velocity $F(\eta, t)$ is obtained for both ramped temperature and isothermal plates and is presented in the following simplified form:

(i) For ramped temperature plate:

$$
\begin{aligned}
F(\eta, t)= & (1 / 2)\left[Q_{0}(\eta, t)-E_{1} Q_{1}(\eta, t)+E_{2} Q_{2}(\eta, t)+E_{3} Q_{3}(\eta, t)+E_{4} Q_{4}(\eta, t)\right. \\
& \left.+H(t-1)\left\{E_{1} Q_{1}(\eta, t-1)-E_{2} Q_{2}(\eta, t-1)-E_{4} Q_{4}(\eta, t-1)\right\}\right],
\end{aligned}
$$

(ii) For isothermal plate:

$$
F(\eta, t)=(1 / 2)\left[R_{0}(\eta, t)-E_{1} R_{1}(\eta, t)+E_{2} R_{2}(\eta, t)+E_{3} R_{3}(\eta, t)+E_{4} R_{4}(\eta, t)\right],
$$

where

$$
\begin{aligned}
Q_{0}(\eta, t)= & f_{1}\left(\eta, t, 1, \lambda_{3}, 0\right), \\
Q_{1}(\eta, t)= & \frac{1}{\alpha_{2} \alpha_{3}\left(\alpha_{2}-\alpha_{3}\right)}\left[\alpha_{3} e^{\alpha_{2} t}\left\{f_{1}\left(\eta, t, 1, \lambda_{3}, \alpha_{2}\right)-f_{1}\left(\eta, t, a, 0, \alpha_{2}\right)\right\}-\alpha_{2} e^{\alpha_{3} t}\left\{f_{1}\left(\eta, t, 1, \lambda_{3}, \alpha_{3}\right)\right.\right. \\
& \left.\left.-f_{1}\left(\eta, t, a, 0, \alpha_{3}\right)\right\}+\left(\alpha_{2}-\alpha_{3}\right)\left\{f_{1}\left(\eta, t, 1, \lambda_{3}, 0\right)-f_{1}(\eta, t, a, 0,0)\right\}\right], \\
Q_{2}(\eta, t)= & \frac{1}{\alpha_{3}^{2}}\left[e^{\alpha_{3} t}\left\{f_{1}\left(\eta, t, 1, \lambda_{3}, \alpha_{3}\right)-f_{1}\left(\eta, t, a, 0, \alpha_{3}\right)\right\}-\alpha_{3}\left\{f_{2}\left(\eta, t, \lambda_{3}, \alpha_{3}\right)-2 f_{3}\left(\eta, t, a, \alpha_{3}\right)\right\}\right], \\
Q_{3}(\eta, t)= & f_{1}\left(\eta, t, 1, \lambda_{3}, 0\right)-f_{1}\left(\eta, t, 1, K_{r}, 0\right), \\
Q_{4}(\eta, t)= & \frac{1}{\alpha_{2}}\left[e^{\alpha_{2} t}\left\{f_{1}\left(\eta, t, 1, \lambda_{3}, \alpha_{2}\right)-f_{1}\left(\eta, t, 1, K_{r}, \alpha_{2}\right)\right\}-\left\{f_{1}\left(\eta, t, 1, \lambda_{3}, \alpha_{3}\right)-f_{1}\left(\eta, t, a, 0, \alpha_{3}\right)\right\}\right], R_{0}(\eta, t)=Q_{0}(\eta, t), \\
R_{1}(\eta, t)= & \frac{1}{\left(\alpha_{2}-\alpha_{3}\right)}\left[e^{\alpha_{2} t}\left\{f_{1}\left(\eta, t, 1, \lambda_{3}, \alpha_{2}\right)-f_{1}\left(\eta, t, a, 0, \alpha_{2}\right)\right\}-e^{\alpha_{3} t}\left\{f_{1}\left(\eta, t, 1, \lambda_{3}, \alpha_{3}\right)-f_{1}\left(\eta, t, a, 0, \alpha_{3}\right)\right\}\right], \\
R_{2}(\eta, t)= & \frac{1}{\alpha_{3}}\left[e^{\alpha_{3} t}\left\{f_{1}\left(\eta, t, 1, \lambda_{3}, \alpha_{3}\right)-f_{1}\left(\eta, t, a, 0, \alpha_{3}\right)\right\}-\left\{f_{1}\left(\eta, t, 1, \lambda_{3}, 0\right)-f_{1}(\eta, t, a, 0,0)\right\}\right], R_{3}(\eta, t)=Q_{3}(\eta, t), \\
R_{4}(\eta, t)= & e^{\alpha_{2} t}\left\{f_{1}\left(\eta, t, 1, \lambda_{3}, \alpha_{2}\right)-f_{1}\left(\eta, t, 1, K_{r}, \alpha_{2}\right)\right\}, \\
\alpha_{1}= & \frac{S_{r} a}{a-1}, \alpha_{2}=\frac{K_{r}}{a-1}, \alpha_{3}=\frac{\lambda_{3}}{a-1}, E_{1}=\frac{\alpha_{1} G_{c}}{a-1}, E_{2}=\frac{G_{r}}{a-1}, E_{3}=\frac{G_{c}}{K_{r}-\lambda_{3}}, E_{4}=\frac{\alpha_{1} G_{c}}{K_{r}-\lambda_{3}} .
\end{aligned}
$$

\section{Shear stress at the plate}

The expressions for shear stress $\tau_{x}$ due to primary flow and shear stress $\tau_{z}$ due to secondary flow at the plate for both ramped temperature and isothermal plates are obtained and are expressed in the following simplified form:

(i) For ramped temperature plate:

$$
\begin{aligned}
\tau_{x}+i \tau_{z}= & \frac{1}{2}\left[S_{0}(0, t)-D_{1} S_{1}(0, t)+D_{2} S_{2}(0, t)+D_{3} S_{3}(0, t)+D_{4} S_{4}(0, t)\right. \\
& \left.+H(t-1)\left\{D_{1} S_{1}(0, t-1)-D_{2} S_{2}(0, t-1)-D_{4} S_{4}(0, t-1)\right\}\right],
\end{aligned}
$$

(ii) For isothermal plate: 


$$
\tau_{x}+i \tau_{z}=\frac{1}{2}\left[P_{0}(0, t)-D_{1} P_{1}(0, t)+D_{2} P_{2}(0, t)+D_{3} P_{3}(0, t)+D_{4} P_{4}(0, t)\right]
$$

where

$$
\begin{aligned}
S_{0}(0, t)= & g_{1}\left(t, 1, \lambda_{3}, 0\right), \\
S_{1}(0, t)= & \frac{1}{\lambda_{2} \lambda_{4}\left(\lambda_{2}-\lambda_{4}\right)}\left[\lambda_{4} e^{\lambda_{2} t}\left\{g_{1}\left(t, 1, \lambda_{2}, \lambda_{3}\right)-g_{1}\left(t, a, \lambda_{2}, 0\right)\right\}-\lambda_{2} e^{\lambda_{4} t}\left\{g_{1}\left(t, 1, \lambda_{3}, \lambda_{4}\right)-g_{1}\left(t, a, 0, \lambda_{4}\right)\right\}\right. \\
& \left.+\left(\lambda_{2}-\lambda_{4}\right)\left\{g_{1}\left(t, 1, \lambda_{3}, 0\right)-g_{1}(t, a, 0,0)\right\}\right], \\
S_{2}(0, t)= & \frac{1}{\lambda_{4}^{2}}\left[e^{\lambda_{4} t}\left\{g_{1}\left(t, 1, \lambda_{3}, \lambda_{4}\right)-g_{1}\left(t, a, 0, \lambda_{4}\right)\right\}-\lambda_{4}\left\{g_{2}\left(t, \lambda_{3}, \lambda_{4}\right)-2 g_{3}\left(t, a, \lambda_{4}\right)\right\}\right], \\
S_{3}(0, t)= & \frac{1}{\lambda_{5}}\left[e^{\lambda_{5} t}\left\{g_{1}\left(t, 1, \lambda_{3}, \lambda_{5}\right)-g_{1}\left(t, S_{c}, K_{r}, \lambda_{5}\right)\right\}-\left\{g_{1}\left(t, 1, \lambda_{3}, 0\right)-g_{1}\left(t, S_{c}, K_{r}, 0\right)\right\}\right], \\
S_{4}(0, t)= & \frac{1}{\lambda_{2} \lambda_{5}\left(\lambda_{2}-\lambda_{5}\right)}\left[\lambda_{5} e^{\lambda_{2} t}\left\{g_{1}\left(t, 1, \lambda_{2}, \lambda_{3}\right)-g_{1}\left(t, S_{c}, \lambda_{2}, K_{r}\right)\right\}-\lambda_{2} e^{\lambda_{5} t}\left\{g_{1}\left(t, 1, \lambda_{3}, \lambda_{5}\right)-g_{1}\left(t, S_{c}, K_{r}, \lambda_{5}\right)\right\}\right. \\
& \left.+\left(\lambda_{2}-\lambda_{5}\right)\left\{g_{1}\left(t, 1, \lambda_{3}, 0\right)-g_{1}\left(t, S_{c}, K_{r}, 0\right)\right\}\right], \\
P_{0}(0, t)= & g_{1}\left(t, 1, \lambda_{3}, 0\right), \\
P_{1}(0, t)= & \frac{1}{\left(\lambda_{2}-\lambda_{4}\right)}\left[e^{\lambda_{2} t}\left\{g_{1}\left(t, 1, \lambda_{2}, \lambda_{3}\right)-g_{1}\left(t, a, \lambda_{2}, 0\right)\right\}-e^{\lambda_{4} t}\left\{g_{1}\left(t, 1, \lambda_{3}, \lambda_{4}\right)-g_{1}\left(t, a, 0, \lambda_{4}\right)\right\}\right], \\
P_{2}(0, t)= & \frac{1}{\lambda_{4}}\left[e^{\lambda_{4} t}\left\{g_{1}\left(t, 1, \lambda_{3}, \lambda_{4}\right)-f_{1}\left(t, a, 0, \lambda_{4}\right)\right\}-\left\{g_{1}\left(t, 1, \lambda_{3}, 0\right)-g_{1}(t, a, 0,0)\right\}\right], \\
P_{3}(\eta, t)= & \frac{1}{\lambda_{5}}\left[e^{\lambda_{5} t}\left\{g_{1}\left(t, 1, \lambda_{3}, \lambda_{5}\right)-g_{1}\left(t, S_{c}, K_{r}, \lambda_{5}\right)\right\}-\left\{g_{1}\left(t, 1, \lambda_{3}, 0\right)-g_{1}\left(t, S_{c}, K_{r}, 0\right)\right\}\right], \\
P_{4}(0, t)= & \frac{1}{\left(\lambda_{2}-\lambda_{5}\right)}\left[e^{\lambda_{2} t}\left\{g_{1}\left(t, 1, \lambda_{2}, \lambda_{3}\right)-g_{1}\left(t, S_{c}, \lambda_{2}, K_{r}\right)\right\}-e^{\lambda_{5} t}\left\{g_{1}\left(t, 1, \lambda_{3}, \lambda_{5}\right)-g_{1}\left(t, S_{c}, K_{r}, \lambda_{5}\right)\right\}\right] . \\
&
\end{aligned}
$$

The expressions for $g_{i}, i=1,2,3$ are defined in Appendix.

\section{Rate of heat transfer at the plate}

The expressions for rate of heat transfer $(\partial T / \partial \eta)_{\eta=0}$ at the plate for both ramped temperature and isothermal plates are obtained and expressed in the following simplified forms:

(i) For ramped temperature plate:

$$
(\partial T / \partial \eta)_{\eta=0}=-2 \sqrt{a / \pi}[\sqrt{t}-H(t-1) \sqrt{t-1}] .
$$

(ii) For isothermal plate:

$$
(\partial T / \partial \eta)_{\eta=0}=-\sqrt{a / \pi t}
$$

It is evident from the expressions (28) and (29) that, for a given time, rate of heat transfer at the plate i.e. $(\partial T / \partial \eta)_{\eta=0}$ is proportional to $\sqrt{a}\left(=\sqrt{P_{r} /\left(1+N_{r}\right)}\right)$ in both the cases, i.e. $(\partial T / \partial \eta)_{\eta=0}$ decreases on increasing $N_{r}$ while it increases on increasing $P_{r}$. Since $P_{r}$ signifies the ratio of momentum diffusivity to thermal diffusivity of the fluid, an increases in thermal diffusivity of the fluid leads to a decrease in $P_{r}$. This implies that thermal radiation and thermal diffusion tend to reduce rate of heat transfer at both the ramped temperature and isothermal plates. Also, it is observed from expressions (28) and (29) that $(\partial T / \partial \eta)_{\eta=0}$ increases for ramped temperature plate whereas it decreases for isothermal plate on increasing time $t$. This implies that rate of heat transfer at the ramped temperature plate is getting enhanced whereas it is getting reduced at the isothermal plate with the progress of time. 


\section{Rate of mass transfer at the plate}

The expressions for rate of mass transfer $(\partial C / \partial \eta)_{\eta=0}$ at the plate for both ramped temperature and isothermal plates are obtained and expressed in the following simplified forms:

(i) For ramped temperature plate:

$$
(\partial C / \partial \eta)_{\eta=0}=-\sqrt{S_{c} / \pi t} e^{-K_{r} t}-\sqrt{S_{c} K_{r}} \operatorname{erf}\left(\sqrt{K_{r} t}\right)+\left(\lambda_{1} / \lambda_{2}\right)\{S(0, t)-H(t-1) S(0, t-1)\},
$$

(ii) For isothermal plate:

$$
\begin{aligned}
(\partial C / \partial \eta)_{\eta=0} & =\lambda_{1} \sqrt{a / \pi t}-\left(1+\lambda_{1}\right) \sqrt{S_{c} / \pi t} e^{-K_{r} t}-\sqrt{S_{c} K_{r}} \operatorname{erf}\left(\sqrt{K_{r} t}\right) \\
& +\lambda_{1}\left\{\sqrt{\lambda_{2} a} \operatorname{erf}\left(\sqrt{\lambda_{2} t}\right)-\sqrt{S_{c}\left(\lambda_{2}+K_{r}\right)} \operatorname{erf}\left(\sqrt{\left(\lambda_{2}+K_{r}\right) t}\right)\right\},
\end{aligned}
$$

where $S(0, t)=\sqrt{S_{c} K_{r}} \operatorname{erf}\left(\sqrt{K_{r} t}\right)+e^{\lambda_{2} t}\left\{\sqrt{\lambda_{2} a} \operatorname{erf}\left(\sqrt{\lambda_{2} t}\right)-\sqrt{S_{c}\left(\lambda_{2}+K_{r}\right)} \operatorname{erf}\left(\sqrt{\left(\lambda_{2}+K_{r}\right) t}\right)\right\}$.

\section{Results and discussion}

In order to study the effects of Hall current, rotation, thermo-diffusion, chemical reaction, thermal radiation and time on the flow-field, the numerical values of primary fluid velocity $u$ and secondary fluid velocity $w$, computed from the analytical solutions (20) and (23) for both ramped temperature and isothermal plates, are displayed graphically versus boundary layer coordinate $\eta$ in Figures 2 to 13 for various values of Hall current parameter $m$, rotation parameter $K^{2}$, Soret number $S_{r}$, chemical reaction parameter $K_{r}$, radiation parameter $N_{r}$ and time $t$ taking magnetic parameter $M^{2}=10$, thermal Grashof number $G_{r}=6$, solutal Grashof number $G_{c}=5$, permeability parameter $K_{1}=0.1$, Prandtl number $P_{r}=0.71$ (ionized air) and Schmidt number $S_{c}=0.6$. Figures 2 and 3 depict the effect of Hall current on the primary and secondary fluid velocities for both ramped temperature and isothermal plates. It revealed from Figures 2 and 3 that, for both ramped temperature and isothermal plates, $u$ and $w$ increase on increasing $m$ throughout the boundary layer region. This implies that, for both ramped temperature and isothermal plates, Hall current tends to accelerate fluid flow in both the primary and secondary flow directions.

It is also observed from Figures 2 and 3 that, for both ramped temperature and isothermal plates, rate of change of fluid velocity in secondary flow direction is faster than that of fluid velocity in primary flow direction. This is justified because Hall current has a tendency to induce secondary flow in the flow-field. Figures 4 and 5 demonstrate the influence of rotation on the primary and secondary fluid velocities for both ramped temperature and isothermal plates. It is evident from Figures 4 and 5 that, for both ramped temperature and isothermal plates, $u$ decreases on increasing $K^{2}$ whereas $w$ increases on increasing $K^{2}$ throughout the boundary layer region. This implies that, for both ramped temperature and isothermal plates, rotation tends to decelerate fluid flow in the primary flow direction whereas it has reverse effect on the fluid flow in the secondary flow direction. This is justifies because Coriolis force, generated due to rotation, tends to suppress fluid flow in the primary flow direction to induce secondary flow in the flow-field. Figures 6 and 7 illustrate the effect of thermo-diffusion on the primary and secondary fluid velocities for both ramped temperature and isothermal plates. It is noticed from Figures 6 and 7 that, for both ramped temperature and isothermal plates, $u$ and $w$ increase on increasing $S_{r}$ throughout the boundary layer region. This implies that, for both ramped temperature and isothermal plates, thermo-diffusion tends to accelerate fluid flow in both the primary and secondary flow directions throughout the boundary layer region.

Figures 8 and 9 present the influence of chemical reaction on the primary and secondary fluid velocities for both ramped temperature and isothermal plates. It is perceived from Figures 8 and 9 that, for both ramped temperature and isothermal plates, $u$ and $w$ decrease on increasing $K_{r}$ throughout the boundary layer region. This implies that, for both ramped temperature and isothermal plates, chemical reaction tends to decelerate fluid flow in both the primary and secondary flow directions throughout the boundary layer region. Figures 10 to 13 display the effects of thermal radiation and time on the primary and secondary fluid velocities for both ramped temperature and isothermal plates. It is observed from Figures 10 to 13 that, for both ramped temperature and isothermal plates, $u$ and $w$ increase on increasing $N_{r}$ and $t$ throughout the boundary layer region. This implies that,

for both ramped temperature and isothermal plates, thermal radiation tends to accelerate fluid flow in both the primary and secondary flow directions throughout the boundary layer region. Also, for both ramped temperature and isothermal plates, fluid flow is getting accelerated with the progress of time in both the primary and secondary flow directions throughout the boundary layer region. Further, it is noticed from Figures 2 to 13 that, for both ramped temperature and isothermal plates, secondary fluid velocity $w$ attains a distinctive maximum value near the surface of the plate and then decreases properly on increasing boundary layer coordinate $\eta$. Moreover, primary and secondary fluid velocities are slower in the case of ramped temperature plate than that of isothermal plate. 
In order to analyze the influence of thermal radiation and time on the temperature field, numerical values of fluid temperature $T$, computed from the analytical solutions (18) and (21) for both ramped temperature and isothermal plate, are shown graphically versus boundary layer coordinate $\eta$ in Figures 14 and 15 for various values of $N_{r}$ and $t$ taking $P_{r}=0.71$. It is evident from Figures 14 and 15 that $T$ increases on increasing $N_{r}$ and $t$ for both ramped temperature and isothermal plates throughout the boundary layer region. This implies that, for both ramped temperature and isothermal plates, thermal radiation tends to enhance fluid temperature throughout the boundary layer region. Also, there is an enhancement in fluid temperature with the progress of time throughout the boundary layer region. Further, it is also observed from Figures 14 and 15 that fluid temperature is higher in the case of isothermal plate than that of ramped temperature plate throughout the boundary layer region.

To study the effects of thermo-diffusion, chemical reaction, thermal radiation and time on the concentration field, numerical values of species concentration $C$, computed from the analytical solutions (19) and (22) for both ramped temperature and isothermal plates, are depicted graphically versus boundary layer coordinate $\eta$ in Figures 16 to 19 for various values of $S_{r}, K_{r}$, $N_{r}$ and $t$ taking $P_{r}=0.71$ and $S_{c}=0.6$. Figure 16 exhibits the effect of thermo-diffusion on the species concentration for both ramped temperature and isothermal plates. It is evident from Figure 16 that, for both ramped temperature and isothermal plates, $C$ increases on increasing $S_{r}$ throughout the boundary layer region. This implies that, for both ramped temperature and isothermal plates, thermo-diffusion tends to enhance species concentration throughout the boundary layer region. Figures 17 and 18 present the influence of chemical reaction and thermal radiation on the species concentration for both ramped temperature and isothermal plates. It is revealed from Figures 17 and 18 that, for both ramped temperature and isothermal plates, $C$ decreases on increasing $K_{r}$ and $N_{r}$ throughout the boundary layer region. This implies that, for both ramped temperature and isothermal plates, chemical reaction and thermal radiation tend to reduce species concentration throughout the boundary layer region. Figure 19 illustrates the effect of time on the species concentration for both ramped temperature and isothermal plates.

It is perceived from Figure 19 that, for both ramped temperature and isothermal plate, $C$ increases on increasing $t$ throughout the boundary layer region. This implies that, for both ramped temperature and isothermal plates, species concentration is getting enhanced with the progress of time for both ramped temperature and isothermal plate throughout the boundary layer region. Further, it is also noticed from Figures 16 to 19 that, species concentration is higher in the case of isothermal plate than that of ramped temperature plate throughout the boundary layer region.

The numerical values of primary shear stress $\tau_{x}$ and secondary shear stress $\tau_{z}$ at the plate, computed from the analytical expressions (26) and (27) for both ramped temperature and isothermal plates, are presented in tabular form in Tables 1 to 6 for various values of $m, K^{2}, S_{r}, K_{r}, N_{r}$ and $t$ taking $M^{2}=10, G_{r}=6, G_{c}=5, K_{1}=0.1, P_{r}=0.71$ and $S_{c}=0.6$. It is evident from Tables 1 and 2 that, $\tau_{x}$ decreases on increasing $m$ whereas it increases on increasing $K^{2}$ for both ramped temperature and isothermal plates. $\tau_{z}$ increases on increasing $m$ and $K^{2}$ for both ramped temperature and isothermal plates. This implies that, for both ramped temperature and isothermal plates, Hall current tends to reduce primary shear stress at the plate whereas it has reverse effect on the secondary shear stress at the plate. Rotation tends to enhance primary as well as secondary shear stress at the plate for both ramped temperature and isothermal plates. It is perceived from Tables 3 and 4 that, $\tau_{x}$ decreases on increasing $S_{r}$ whereas it increases on increasing $K_{r}$ for both ramped temperature and isothermal plates. $\tau_{z}$ increases on increasing $S_{r}$ whereas it decreases on increasing $K_{r}$ for both ramped temperature and isothermal plates. This implies that, for both ramped temperature and isothermal plates, thermo-diffusion tends to reduce primary shear stress at the plate whereas it has a reverse effect on the secondary shear stress at the plate. Chemical reaction tends to enhance primary shear stress at the plate whereas it has a reverse effect on the secondary shear stress at the plate for both ramped temperature and isothermal plates.

It is revealed from Tables 5 and 6 that, $\tau_{x}$ decreases on increasing $N_{r}$ and $t$ whereas $\tau_{z}$ increases on increasing $N_{r}$ and $t$ for both ramped temperature and isothermal plates. This implies that, for both ramped temperature and isothermal plates, thermal radiation tends to reduce primary shear stress at the plate whereas it has a reverse effect on the secondary shear stress at the plate. Primary shear stress at the plate is getting reduced whereas secondary shear stress at the plate is getting enhanced with the progress of time for both ramped temperature and isothermal plates.

The numerical values of rate of mass transfer at the plate i.e. $(\partial C / \partial \eta)_{\eta=0}$, computed from the analytical expression (30) and (31) for both ramped temperature and isothermal plates, are presented in Tables 7 and 8 for various values of $S_{r}, K_{r}, N_{r}$ and $t$ taking $P_{r}=0.71$ and $S_{c}=0.6$. It is noticed from Table 7 that rate of mass transfer at the plate i.e. $(\partial C / \partial \eta)_{\eta=0}$ decreases on increasing $S_{r}$ whereas it increases on increasing $K_{r}$ for both ramped temperature and isothermal plates. This implies that, for both ramped temperature and isothermal plates, thermo-diffusion tends to reduce rate of mass transfer at the plate whereas chemical reaction has a reverse effect on the rate of mass transfer at the plate. It is evident from Table 8 that $(\partial C / \partial \eta)_{\eta=0}$ increases on increasing $N_{r}$

whereas it decreases on increasing $t$ for both ramped temperature and isothermal plates. This implies that, for both ramped temperature and isothermal plates, thermal radiation tends to enhance rate of mass transfer at the plate whereas rate of mass transfer at the plate is getting reduced with the progress of time. 


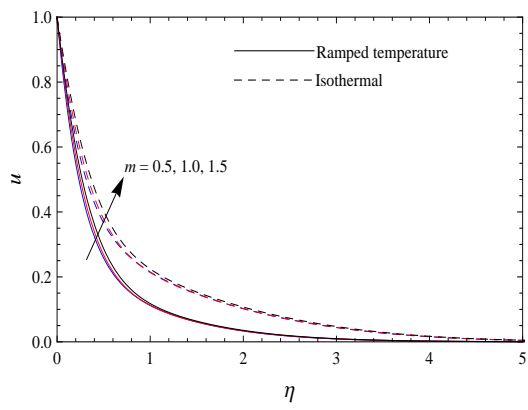

Figure 2 Primary velocity profiles when $K^{2}=4, S_{r}=2, K_{r}=2.5, N_{r}=2$ and $t=0.5$.

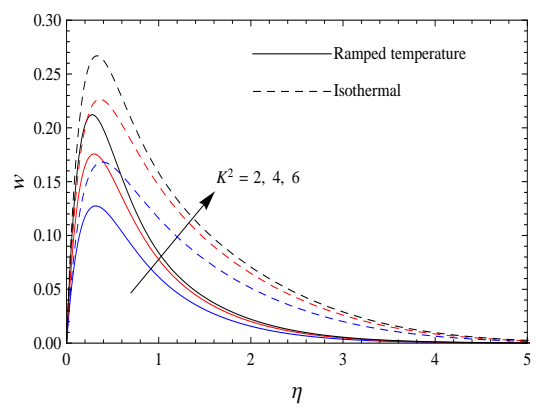

Figure 5 Secondary velocity profiles when $m=0.5, S_{r}=2, K_{r}=2.5, N_{r}=2$ and $t=0.5$.

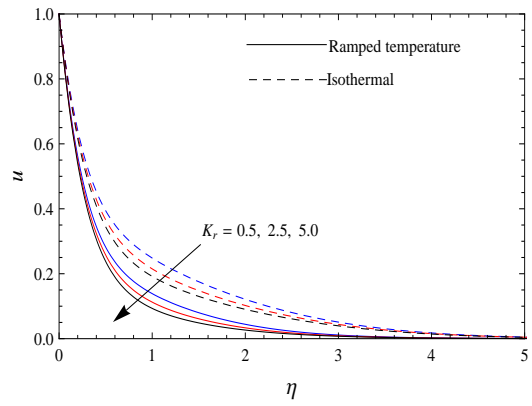

Figure 8 Primary velocity profiles when $m=0.5, K^{2}=4, S_{r}=2, N_{r}=2$ and $t=0.5$.

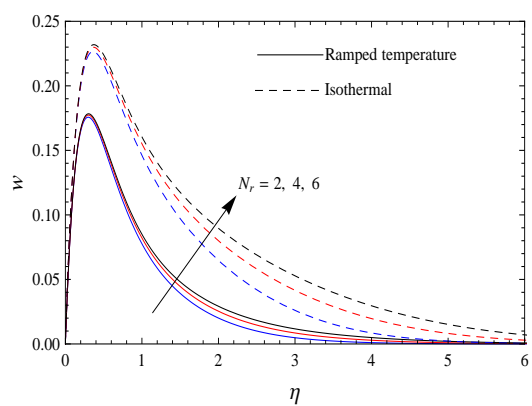

Figure 11 Secondary velocity profiles when $m=0.5, K^{2}=4, S_{r}=2, K_{r}=2.5$ and $t=0.5$.

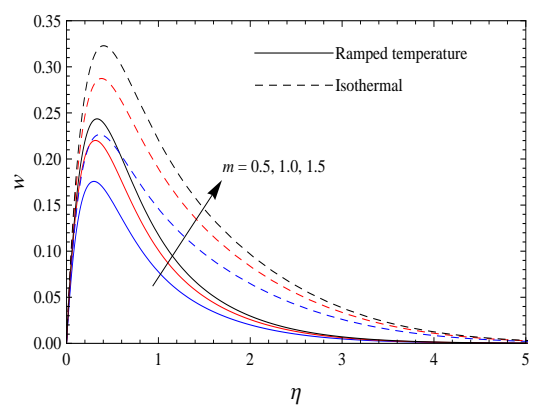

Figure 3 Secondary velocity profiles when $K^{2}=4, S_{r}=2, K_{r}=2.5, N_{r}=2$ and $t=0.5$.

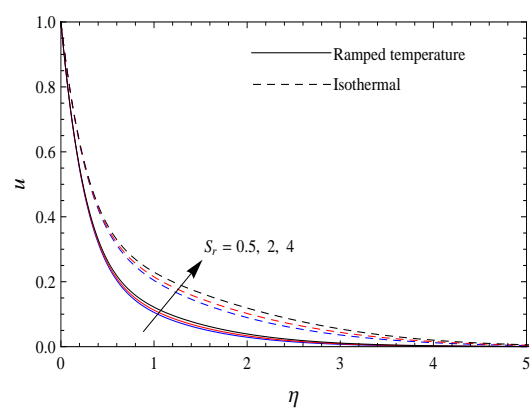

Figure 6 Primary velocity profiles when $m=0.5, K^{2}=4, K_{r}=2.5, N_{r}=2$ and $t=0.5$.

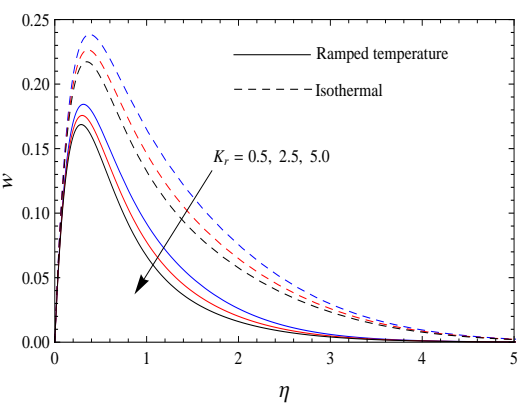

Figure 9 Secondary velocity profiles when $m=0.5, K^{2}=4, S_{r}=2, N_{r}=2$ and $t=0.5$.

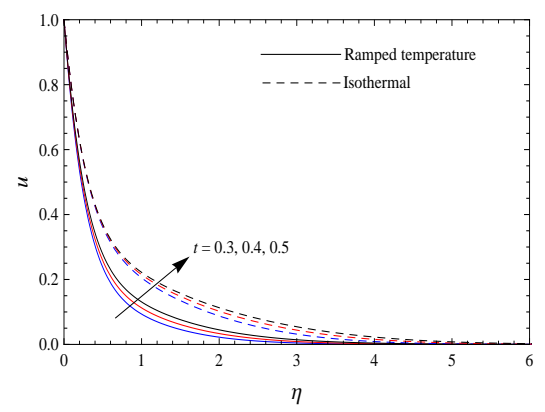

Figure 12 Primary velocity profiles when $m=0.5, K^{2}=4, S_{r}=2, K_{r}=2.5$ and $N_{r}=2$.

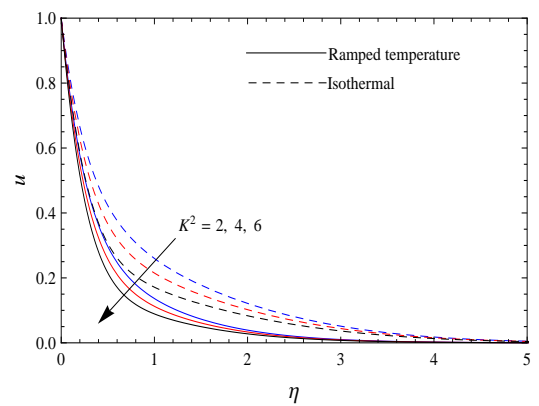

Figure 4 Primary velocity profiles when $m=0.5, S_{r}=2, K_{r}=2.5, N_{r}=2$ and $t=0.5$.

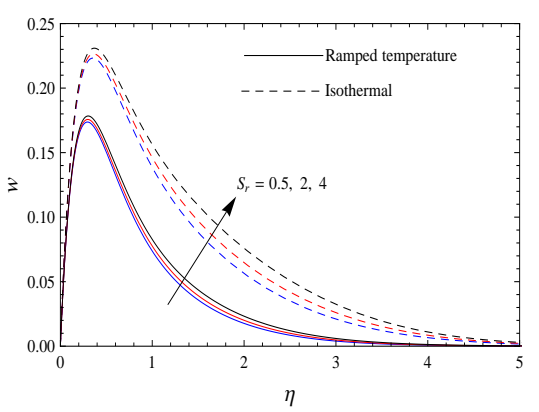

Figure 7 Secondary velocity profiles when $m=0.5, K^{2}=4, K_{r}=2.5, N_{r}=2$ and $t=0.5$.

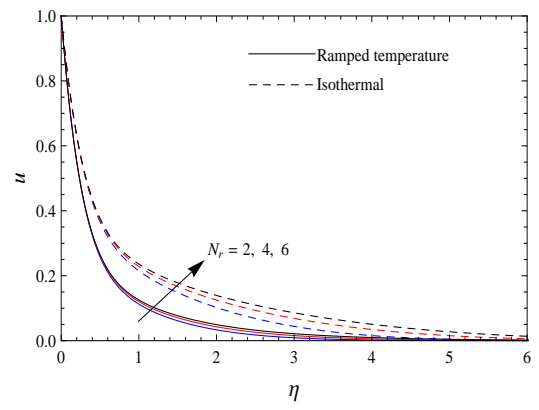

Figure 10 Primary velocity profiles when $m=0.5, K^{2}=4, S_{r}=2, K_{r}=2.5$ and $t=0.5$.

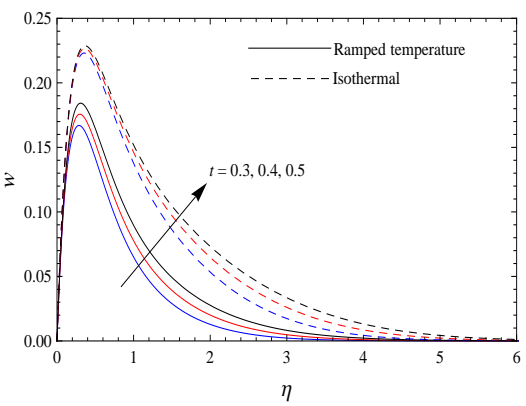

Figure 13 Secondary velocity profiles when $m=0.5, K^{2}=4, S_{r}=2, K_{r}=2.5$ and $N_{r}=2$. 


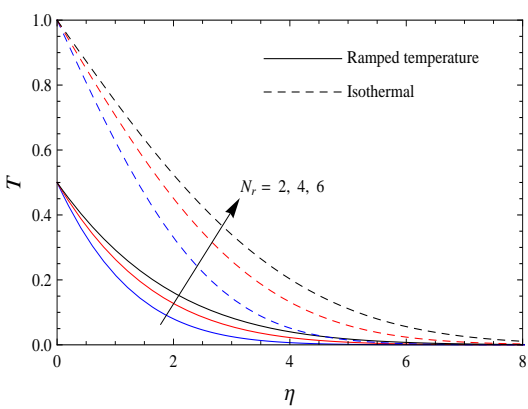

Figure 14 Temperature profiles when $t=0.5$.

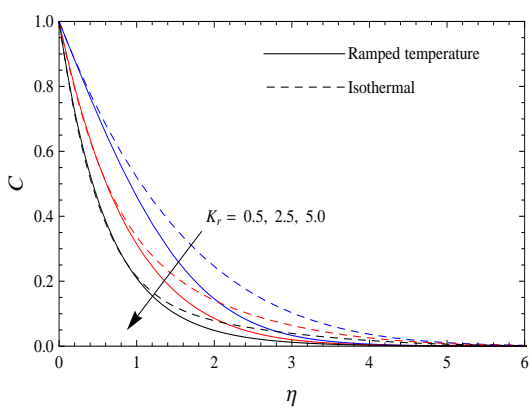

Figure 17 Concentration profiles when $S_{r}=2$, $N_{r}=2$ and $t=0.5$.

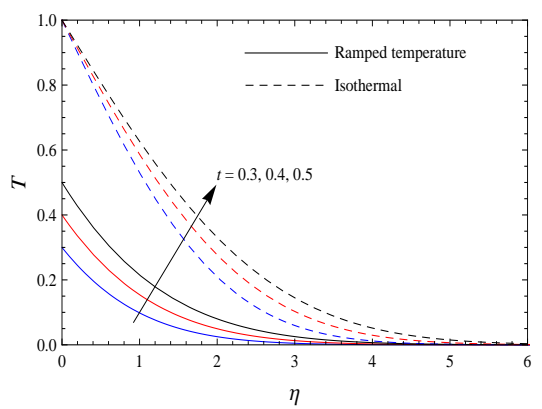

Figure 15 Temperature profiles when $N_{r}=2$.

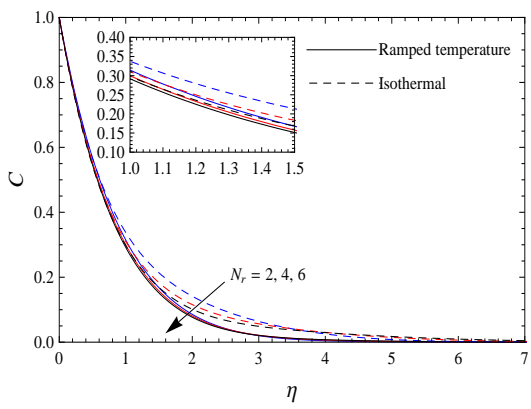

Figure 18 Concentration profiles when $S_{r}=2$, $K_{r}=2.5$ and $t=0.5$.

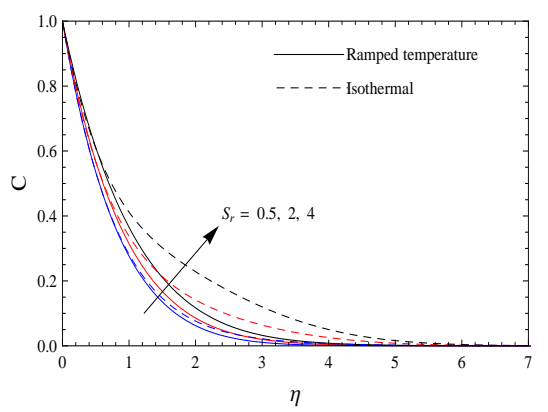

Figure 16 Concentration profiles when $K_{r}=2.5$, $N_{r}=2$ and $t=0.5$.

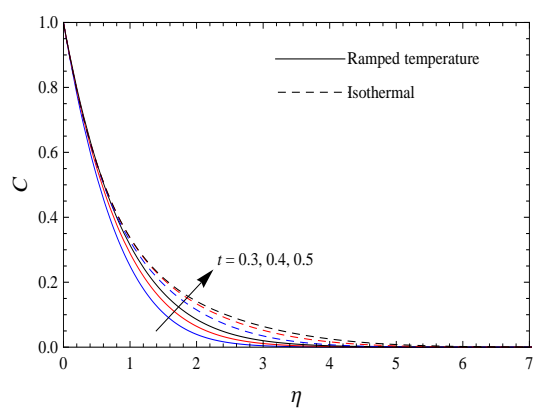

Figure 19 Concentration profiles when $S_{r}=2$, $K_{r}=2.5$ and $N_{r}=2$.
Table 1 Shear stress at ramped temperature plate when $S_{r}=2$, $K_{r}=2.5, N_{r}=2$ and $t=0.5$.

\begin{tabular}{|c|c|c|c|c|c|c|}
\hline \multirow{2}{*}{$\begin{array}{l}K^{2} \rightarrow \\
m \downarrow\end{array}$} & \multicolumn{3}{|c|}{$-\tau_{x}$} & \multicolumn{3}{c|}{$\tau_{z}$} \\
\cline { 2 - 7 } & 2 & 4 & 6 & 2 & 4 & 6 \\
\hline 0.5 & 2.913 & 3.086 & 3.293 & 1.153 & 1.668 & 2.130 \\
\hline 1.0 & 2.542 & 2.772 & 3.027 & 1.425 & 1.955 & 2.417 \\
\hline 1.5 & 2.239 & 2.504 & 2.792 & 1.476 & 2.034 & 2.508 \\
\hline
\end{tabular}

Table 3 Shear stress at ramped temperature plate when $m=0.5$, $K^{2}=4, N_{r}=2$ and $t=0.5$.

\begin{tabular}{|c|c|c|c|c|c|c|}
\hline \multirow{2}{*}{$\begin{array}{l}K_{r} \rightarrow \\
S_{r} \downarrow\end{array}$} & \multicolumn{3}{|c|}{$-\tau_{x}$} & \multicolumn{3}{c|}{$\tau_{z}$} \\
\hline 0.5 & & 2.5 & 5 & 0.5 & 2.5 & 5 \\
\hline 2 & 3.037 & 3.101 & 3.158 & 1.690 & 1.661 & 1.635 \\
\hline 4 & 3.019 & 3.086 & 3.146 & 1.699 & 1.668 & 1.641 \\
\hline
\end{tabular}

Table 5 Shear stress at ramped temperature plate when $m=0.5$, $K^{2}=4, S_{r}=2$ and $K_{r}=2.5$.

\begin{tabular}{|c|c|c|c|c|c|c|}
\hline \multirow{2}{*}{$\begin{array}{c}t \rightarrow \\
N_{r} \downarrow\end{array}$} & \multicolumn{3}{|c|}{$-\tau_{x}$} & \multicolumn{3}{c|}{$\tau_{z}$} \\
\cline { 2 - 7 } & 0.3 & 0.4 & 0.5 & 0.3 & 0.4 & 0.5 \\
\hline 2 & 3.331 & 3.207 & 3.086 & 1.592 & 1.632 & 1.668 \\
\hline 4 & 3.324 & 3.199 & 3.077 & 1.596 & 1.636 & 1.673 \\
\hline 6 & 3.320 & 3.194 & 3.071 & 1.598 & 1.639 & 1.677 \\
\hline
\end{tabular}

Table 2 Shear stress at isothermal plate when $S_{r}=2, K_{r}=2.5$, $N_{r}=2$ and $t=0.5$.

\begin{tabular}{|c|c|c|c|c|c|c|}
\hline \multirow{2}{*}{$\begin{array}{c}K^{2} \rightarrow \\
m \downarrow\end{array}$} & \multicolumn{3}{|c|}{$-\tau_{x}$} & \multicolumn{3}{|c|}{$\tau_{z}$} \\
\cline { 2 - 7 } & 2 & 4 & 6 & 2 & 4 & 6 \\
\hline 0.5 & 2.244 & 2.464 & 2.719 & 1.299 & 1.862 & 2.355 \\
\hline 1.0 & 1.842 & 2.136 & 2.451 & 1.625 & 2.201 & 2.686 \\
\hline 1.5 & 1.502 & 1.847 & 2.204 & 1.705 & 2.309 & 2.803 \\
\hline
\end{tabular}

Table 4 Shear stress at isothermal plate when $m=0.5, K^{2}=4$, $N_{r}=2$ and $t=0.5$.

\begin{tabular}{|c|c|c|c|c|c|c|}
\hline \multirow{2}{*}{$\begin{array}{c}K_{r} \rightarrow \\
S_{r} \downarrow\end{array}$} & \multicolumn{3}{|c|}{$-\tau_{x}$} & \multicolumn{3}{c|}{$\tau_{z}$} \\
\cline { 2 - 7 } & 0.5 & 2.5 & 5 & 0.5 & 2.5 & 5 \\
\hline 0.5 & 2.411 & 2.477 & 2.535 & 1.883 & 1.853 & 1.827 \\
\hline 2 & 2.389 & 2.464 & 2.527 & 1.898 & 1.862 & 1.832 \\
\hline 4 & 2.359 & 2.446 & 2.517 & 1.918 & 1.874 & 1.840 \\
\hline
\end{tabular}

Table 6 Shear stress at isothermal plate when $m=0.5$, $K^{2}=4, S_{r}=2$ and $K_{r}=2.5$.

\begin{tabular}{|c|c|c|c|c|c|c|}
\hline \multirow{2}{*}{$\begin{array}{c}t \rightarrow \\
N_{r} \downarrow\end{array}$} & \multicolumn{3}{|c|}{$-\tau_{x}$} & \multicolumn{3}{|c|}{$\tau_{z}$} \\
\hline 2 & 0.3 & 0.4 & 0.5 & 0.3 & 0.4 & 0.5 \\
\hline 2 & 2.492 & 2.475 & 2.464 & 1.835 & 1.852 & 1.862 \\
\hline 4 & 2.477 & 2.460 & 2.450 & 1.846 & 1.865 & 1.871 \\
\hline 6 & 2.467 & 2.452 & 2.443 & 1.853 & 1.868 & 1.877 \\
\hline
\end{tabular}


Table 7 Rate of mass transfer at the plate when $N_{r}=2$ and $t=0.5$.

\begin{tabular}{|c|c|c|c|c|c|c|}
\hline \multirow{2}{*}{$K_{r} \rightarrow$} & \multicolumn{6}{|c|}{$-(\partial C / \partial \eta)_{\eta=0}$} \\
\cline { 2 - 7 } & \multicolumn{3}{|c|}{ Ramped temperature } & \multicolumn{3}{c|}{ Isothermal plate } \\
\cline { 2 - 7 }$S_{r} \downarrow$ & 0.5 & 2.5 & 5.0 & 0.5 & 2.5 & 5.0 \\
\hline 0.5 & & & & & & \\
\hline 2 & 0.724 & 1.226 & 1.708 & 0.728 & 1.240 & 1.726 \\
\hline 4 & 0.595 & 1.118 & 1.617 & 0.612 & 1.173 & 1.689 \\
\hline
\end{tabular}

Table 8 Rate of mass transfer at the plate when $S_{r}=2$ and $K_{r}=2.5$.

\begin{tabular}{|c|c|c|c|c|c|c|}
\hline \multirow[b]{3}{*}{$\begin{array}{l}t \rightarrow \\
N_{r} \downarrow\end{array}$} & \multicolumn{6}{|c|}{$-(\partial C / \partial \eta)_{\eta=0}$} \\
\hline & \multicolumn{3}{|c|}{$\begin{array}{c}\text { Ramped temperature } \\
\text { plate }\end{array}$} & \multicolumn{3}{|c|}{ Isothermal plate } \\
\hline & 0.3 & 0.4 & 0.5 & 0.3 & 0.4 & 0.5 \\
\hline 2 & 1.210 & 1.152 & 1.118 & 1.182 & 1.173 & 1.173 \\
\hline 4 & 1.253 & 1.20 & 1.170 & 1.237 & 1.216 & 1.208 \\
\hline 6 & 1.273 & 1.222 & 1.193 & 1.263 & 1.235 & 1.223 \\
\hline
\end{tabular}

\section{Conclusion}

Theoretical investigation of Soret and Hall effects on unsteady hydromagnetic free convection heat and mass transfer flow of a viscous, incompressible, electrically conducting and optically thick radiating fluid past an impulsively moving infinite vertical plate with ramped temperature through a uniform porous medium in a rotating system in the presence of first order chemical reaction is carried out. Significant findings within the boundary layer region are as follows:

For both ramped temperature and isothermal plates-

- Hall current, thermo-diffusion, thermal radiation tend to accelerate fluid flow in both the primary and secondary flow whereas chemical reaction has a reverse effect on it.

- Rotation tends to decelerate fluid flow in the primary flow direction whereas it has reverse effect on the fluid flow in the secondary flow direction.

- Fluid flow is getting accelerated with the progress of time in both the primary and secondary flow directions.

- Thermal radiation tends to enhance fluid temperature and there is an enhancement in fluid temperature with the progress of time throughout the boundary layer region.

- Thermo-diffusion tends to enhance species concentration whereas chemical reaction and thermal radiation have reverse effect on it. Species concentration is getting enhanced with the progress of time throughout the boundary layer region.

- Hall current, thermo-diffusion and thermal radiation tend to reduce primary shear stress at the plate whereas these agencies have reverse effect on the secondary shear stress at the plate. Rotation tends to enhance primary as well as secondary shear stress at the plate.

- Chemical reaction tends to enhance primary shear stress at the plate whereas it has a reverse effect on the secondary shear stress at the plate.

- Primary shear stress at the plate is getting reduced whereas secondary shear stress at the plate is getting enhanced with the progress of time.

- Thermo-diffusion tends to reduce rate of mass transfer at the plate whereas chemical reaction has a reverse effect on it. Thermal radiation tends to enhance rate of mass transfer at the plate whereas rate of mass transfer at the plate is getting reduced with the progress of time.

\section{Acknowledgements}

Authors are grateful to Indian School of Mines, Dhanbad for providing financial support and good research facility to carry out this work.

\section{References}

Alam, M. M. and Sattar, M. A., 2000. Unsteady MHD free convection and mass transfer flow in a rotating system with Hall current, viscous dissipation and Joule heating, J. Energy Heat Mass Transf., Vol. 22, pp. 31-39.

Azzam, G. E. A., 2002. Radiation effects on the MHD mixed free-forced convective flow past a semi-infinite moving vertical plate for high temperature differences, Phys. Scr., Vol. 66, No. 1, pp. 71-76.

Bakr, A. A., 2011. Effects of chemical reaction on MHD free convection and mass transfer flow of a micropolar fluid with oscillatory plate velocity and constant heat source in a rotating frame of reference, Comm. Nonlinear Sci. Numer. Simulat., Vol. 16, No. 2, pp. 698-710.

Barik, R. N. and Dash, G. C., 2014. Thermal radiation effect on an unsteady Magnetohydrodynamic flow past inclined porous heated plate in the presence of chemical reaction and viscous dissipation, Appl. Math. Comput., Vol. 226, No. 1, pp. 423-434.

Barik, R. N., Dash, G. C. and Kar, M., 2014. Unsteady free convective MHD flow and mass transfer through porous medium in a rotating system with fluctuating heat source/sink and chemical reaction, J. Appl. Analys. Comput. Vol. 4, No. 3, pp. 231-244.

Chandran, P., Sacheti, N. C. and Singh, A. K., 2005. Natural convection near a vertical plate with ramped wall temperature, Heat Mass Transf., Vol. 41, No. 5, pp. 459-464. 
Cramer, K. R. and Pai, S. I., 1973. Magnetofluid Dynamics for Engineers and Applied Physicists, New York: McGraw Hill Book Company.

Das, K., 2011. Effect of chemical reaction and thermal radiation on heat and mass transfer flow of MHD micropolar fluid in a rotating frame of reference, Int. J. Heat Mass Transf., Vol. 54, pp. 3505-3513.

Das, S. S., Satapathy, A., Das, J. K. and Panda, J. P., 2009. Mass transfer effects on MHD flow and heat transfer past a vertical porous plate through a porous medium under oscillatory suction and heat source, Int. J. Heat Mass Transf., Vol. 52, pp. 59625969.

Eckert, E. R. G. and Drake, R. M., 1972. Analysis of Heat and Mass Transfer, McGraw Hill.

Eldabe, N. T. M., Elbashbeshy, E. M. A., Hasanin, W. S. A. and Elsaid, E. M., 2011. Unsteady motion of MHD viscous incompressible fluid with heat and mass transfer through porous medium near a moving vertical plate, Int. J. Energy Tech., Vol. 3, No. 35, pp. 1-11.

Ferdows, M., Kaino, K. and Crepeau, J. C., 2008. MHD free convection and mass transfer flow in a porous media with simultaneous rotating fluid, Int. J. Dyn. Fluids, Vol. 4, No. 1, pp. 69-82.

Ibrahim, F. S., Elaiw, A. M. and Bakr, A. A., 2008. Effect of the chemical reaction and radiation absorption on the unsteady MHD free convection flow past a semi infinite vertical permeable moving plate with heat source and suction, Comm. Nonlinear Sci. Numer. Simulat., Vol. 13, No. 6, pp. 1056-1066.

Ibrahim, F. S., Hassanien, I. A. and Bakr, A. A., 2004. Unsteady Magnetohydrodynamic micropolar fluid flow and heat transfer over a vertical porous plate through a porous medium in the presence of thermal and mass diffusion with a constant heat source, Canad. J. Phys., Vol. 82, No. 10, pp. 775-790.

Jha, B. K. and Singh, A. K., 1990. Soret effects on free-convection and mass transfer flow in the Stokes problem for an infinite vertical plate. Astrophys. Space Sci., Vol. 173, No. 2, pp. 251-255.

Jha, B. K., 1991. MHD free-convection and mass-transform flow through a porous medium, Astrophys. Space Sci., Vol. 175, No. 2, pp. 283-289.

Kafoussias, N. G., 1992. MHD thermal-diffusion effects on free-convective and mass-transfer flow over an infinite vertical moving plate. Astrophys. Space Sci., Vol. 192, No. 1, pp. 11-19.

Kamel, M. H., 2001. Unsteady MHD convection through porous medium with combined heat and mass transfer with heat source/sink, Energy Conversion and Management, Vol. 42, No. 4, pp. 393-405.

Lee, S. and Yovanovich, M. M., (1991). Laminar natural convection from a vertical plate with a step change in wall temperature, J. Heat Transf., Vol. 113, No. 2, pp. 501-504.

Mahmoud, M. A. A., 2009. Thermal radiation effect on unsteady MHD free convection flow past a vertical plate with temperaturedependent viscosity, Canad. J. Chem. Eng., Vol. 87, No. 1, pp. 47-52.

Malhotra, C. P., Mahajan, R. L., Sampath, W. S., Barth, K. L. and Enzenroth, R. A., 2006. Control of temperature uniformly during manufacture of stable thin-film photo-voltaic devices, Int. J. Heat Mass Transf., Vol. 49, pp. 2840-2850.

Mbeledogu, I. U. and Ogulu, A., 2007. Heat and mass transfer of an unsteady MHD natural convection flow of a rotating fluid past a vertical porous flat plate in the presence of radiative heat transfer, Int. J. Heat and Mass Transf., Vol. 50, pp. 1902-1908.

Mohamed, R. A. and Abo-Dahab, S. M., 2009. Influence of chemical reaction and thermal radiation on the heat and mass transfer in MHD micropolar flow over a vertical moving porous plate in a porous medium with heat generation, Int. J. Thermal Sci., Vol. 48, No. 9, pp. 1800-1813.

Nandkeolyar, R., Das, M. and Sibanda, P., 2013. Unsteady hydromagnetic heat and mass transfer flow of a heat radiating and chemically reactive fluid past a flat porous plate with ramped wall temperature, Math. Prob. Eng., 2013, Article ID 381806, doi:10.1155/2013/381806.

Nanousis, N., 1992. Thermal-diffusion effects on MHD free-convective and mass-transfer flow past a moving infinite vertical plate in a rotating fluid, Astrophys. Space Sci., 191(2), pp. 313-322.

Ogulu, A., Makinde, O. D., 2008. Unsteady hydromagnetic free convection flow of a dissipative and radiating fluid past a vertical plate with constant heat flux, Chem. Eng. Comm., Vol. 196, No. 4, pp. 454-462.

Osman, A. N. A., Abo-Dahab, S. M. and Mohamed, R. A., 2011. Analytical solution of thermal radiation and chemical reaction effects on unsteady MHD convection through porous media with heat source/sink," Math. Prob. Eng., 2011, Article ID 205181, doi:10.1155/2011/205181.

Pal, D. and Talukdar, B., 2012. Influence of fluctuating thermal and mass diffusion on unsteady MHD buoyancy-driven convection past a vertical surface with chemical reaction and Soret effects, Comm. Nonlinear Sci. Numer. Simulat., Vol. 17, No. 4, pp.1597-1614.

Pal, D. and Talukdar, B., 2011. Combined effects of Joule heating and chemical reaction on unsteady Magnetohydrodynamic mixed convection of a viscous dissipating fluid over a vertical plate in porous media with thermal radiation, Math. Comput. Modell., Vol. 54, pp. 3016-3036.

Reddy, B. P. and Rao, J. A., 2011. Radiation and thermal diffusion effects on an unsteady MHD free convection mass-transfer flow past an infinite vertical porous plate with the Hall current and a heat source, J. Eng. Phys. Thermophys., Vol. 84, No. 6, pp. 1369-1378. 
Seth, G. S., Nandkeolyar, R. and Ansari, M. S., 2013. Effects of thermal radiation and rotation on unsteady hydromagnetic free convection flow past an impulsively moving vertical plate with ramped temperature in a porous medium, J. Appl. Fluid Mech. Vol. 6, No. 1, pp. 27-38.

Seth, G. S., Sarkar, S. and Hussain, S. M., 2014. Effects of Hall current, radiation and rotation on natural convection heat and mass transfer flow past a moving vertical plate, Ain Shams Eng. J., Vol. 5, No. 2, pp. 489-503.

Seth, G. S., Ansari, M. S. and Nandkeolyar, R., 2011. MHD natural convection flow with radiative heat transfer past an impulsively moving plate with ramped wall temperature, Heat Mass Transf., Vol. 47, No. 5, pp. 551-561.

Seth, G. S., Hussain, S. M., Sarkar, S., 2015. Hydromagnetic natural convection flow with heat and mass transfer of a chemically reacting and heat absorbing fluid past an accelerated moving vertical plate with ramped temperature and ramped surface concentration through a porous medium, J. Egyptian Math. Soc., Vol. 23, pp. 197-207.

Singh, K. D. and Kumar, R., .2010. Effects of chemical reactions on unsteady MHD free convection and mass transfer for flow past a hot vertical porous plate with heat generation/absorption through porous medium, Ind. J. Phys., Vol. 84, No. 1, pp. 93106.

Sparrow, E. M. and Cess, R. D., 1966. Radiation Heat Transfer. Belmont, Calif.: Brooks/Cole.

Suneetha, S., Reddy, N. B. and Prasad, V. R., 2009. Radiation and mass transfer effects on MHD free convection flow past an impulsively started isothermal vertical plate with dissipation, Thermal Sci., Vol. 13, No. 2, pp.171-181.

Sutton, G. W. and Sherman, A., 1965. Engineering Magnetohydrodynamics, New York: McGraw-Hill.

Symeonidis, V., Karniadakis, G. E. and Caswell, B., 2006. Schmidt number effects in simulation of polymer dissipative particle dynamics, The J. Chem. Phys., Vol. 125, No. 18, Article ID: 184902, http://dx.doi.org/10.1063/1.2360274.

Takhar, H. S., Gorla, R. S. R. and Soundalgekar, V. M., 1996. Short communication radiation effects on MHD free convection flow of a gas past a semi-infinite vertical plate, Int. J. Numer. Methods Heat Fluid Flow, Vol. 6, No. 2, pp. 77-83.

Tokis, J. N., 1988. Free convection and mass transfer effects on the Magnetohydrodynamic flows near a moving plate in a rotating medium, Astrophys. Space Sci., Vol. 144, pp. 291-301.

\section{Appendix}

$$
\begin{aligned}
& f_{1}\left(c_{1}, c_{2}, c_{3}, c_{4}, c_{5}\right)=e^{c_{1} \sqrt{c_{3}\left(c_{4}+c_{5}\right)}} \operatorname{erfc}\left(\frac{c_{1}}{2} \sqrt{\frac{c_{3}}{c_{2}}}+\sqrt{\left(c_{4}+c_{5}\right) c_{2}}\right)+e^{-c_{1} \sqrt{c_{3}\left(c_{4}+c_{5}\right)}} \operatorname{erfc}\left(\frac{c_{1}}{2} \sqrt{\frac{c_{3}}{c_{2}}}-\sqrt{\left(c_{4}+c_{5}\right) c_{2}}\right) \\
& f_{2}\left(c_{1}, c_{2}, c_{3}, c_{4}\right)=\left(c_{2}+\frac{1}{c_{4}}+\frac{c_{1}}{2 \sqrt{c_{3}}}\right) e^{c_{1} \sqrt{c_{3}}} \operatorname{erfc}\left(\frac{c_{1}}{2 \sqrt{c_{2}}}+\sqrt{c_{2} c_{3}}\right)+\left(c_{2}+\frac{1}{c_{4}}-\frac{c_{1}}{2 \sqrt{c_{3}}}\right) e^{-c_{1} \sqrt{c_{3}}} \operatorname{erfc}\left(\frac{c_{1}}{2 \sqrt{c_{2}}}-\sqrt{c_{2} c_{3}}\right) \\
& f_{3}\left(c_{1}, c_{2}, c_{3}, c_{4}, c_{5}\right)=\left(c_{2}+\frac{1}{c_{4}}+\frac{c_{1}^{2} c_{3}}{2}\right) \operatorname{erfc}\left(\frac{c_{1}}{2} \sqrt{\frac{c_{3}}{c_{2}}}\right)-c_{1} \sqrt{\frac{c_{2} c_{3}}{c_{5}}} e^{-\frac{c_{1}^{2} c_{3}}{4 c_{2}}} . \\
& g_{1}\left(d_{1}, d_{2}, d_{3}, d_{4}\right)=-2 \sqrt{\frac{d_{2}}{\pi d_{1}}} e^{-d_{1}\left(d_{3}+d_{4}\right)}-2 \sqrt{d_{2}\left(d_{3}+d_{4}\right)} \operatorname{erf}\left(\sqrt{d_{1}\left(d_{3}+d_{4}\right)}\right), \\
& g_{2}\left(d_{1}, d_{2}, d_{3}\right)=-\frac{1}{d_{3}}\left[\frac{2}{\sqrt{\pi d_{1}}}\left(1+d_{1} d_{3}\right) e^{-d_{1} d_{2}}+\frac{1}{\sqrt{d_{2}}}\left\{d_{3}+2 d_{2}\left(1+d_{1} d_{3}\right)\right\} \operatorname{erf}\left(\sqrt{d_{1} d_{2}}\right)\right] \\
& g_{3}\left(d_{1}, d_{2}, d_{3}\right)=-\sqrt{\frac{d_{2}}{\pi}}\left[\sqrt{d_{1}}-\sqrt{\frac{1}{d_{1}}}\left(d_{2}+\frac{1}{d_{3}}\right)\right] .
\end{aligned}
$$

\section{Biographical notes}

G. S. Seth, B. Kumbhakar and S. Sarkar are all of the Department of Applied Mathematics, Indian School of Mines, Dhanbad-826004, India

Received March 2015

Accepted May 2015

Final acceptance in revised form June 2015 